Service social

\title{
Chômage de longue durée, transmissions générationnelles et modèles normatifs
}

\section{Nathalie Burnay}

Volume 55, numéro 1, 2009

URI : https://id.erudit.org/iderudit/029489ar

DOI : https://doi.org/10.7202/029489ar

Aller au sommaire du numéro

Éditeur(s)

École de service social de l'Université Laval

ISSN

1708-1734 (numérique)

Découvrir la revue

Citer cet article

Burnay, N. (2009). Chômage de longue durée, transmissions générationnelles et modèles normatifs. Service social, 55(1), 47-65. https://doi.org/10.7202/029489ar

\section{Résumé de l'article}

Depuis les enquêtes de Lazarsfeld dans les années 1930, de nombreux scientifiques ont mis en évidence les conséquences économiques, psychiques et relationnelles qu'engendre une expérience de chômage. Mais plus rares sont les études consacrées au devenir des enfants. Pourtant, la crise qui affecte les pays occidentaux depuis la moitié des années 1970 n'est pas sans poser la question de la transmission des cadres normatifs dans un contexte familial de chômage de très longue durée. À travers l'analyse d'entretiens croisés au sein de familles, cet article va tenter de démêler les fils complexes des logiques de transmission générationnelle dans un tel contexte de déstructuration du rapport au travail et à l'emploi. Deux clés interprétatives permettront de mieux cerner les enjeux personnels, familiaux et sociaux d'une crise qui perdure : la présence ou non d'une souffrance parentale et la possibilité pour le jeune de nouer des rapports de confiance interpersonnels. 


\title{
Chômage de longue durée, transmissions générationnelles et modèles normatifs
}

\author{
Nathalie BURNAY \\ Docteur en Sociologie \\ Chargée de cours
}

Facultés universitaires Notre-Dame de la Paix de Namur (FUNDP), Belgique

Université catholique de Louvain, Belgique

\begin{abstract}
Depuis les enquêtes de Lazarsfeld dans les années 1930, de nombreux scientifiques ont mis en évidence les conséquences économiques, psychiques et relationnelles qu'engendre une expérience de chômage. Mais plus rares sont les études consacrées au devenir des enfants. Pourtant, la crise qui affecte les pays occidentaux depuis la moitié des années 1970 n’est pas sans poser la question de la transmission des cadres normatifs dans un contexte familial de chômage de très longue durée. À travers l'analyse d'entretiens croisés au sein de familles, cet article va tenter de démêler les fils complexes des logiques de transmission générationnelle dans un tel contexte de déstructuration du rapport au travail et à l'emploi. Deux clés interprétatives permettront de mieux cerner les enjeux personnels, familiaux et sociaux d'une crise qui perdure : la présence ou non d'une souffrance parentale et la possibilité pour le jeune de nouer des rapports de confiance interpersonnels.
\end{abstract}

Mots clés : Chômage de longue durée, transmission générationnelle, confiance, souffrance

Since Lazarsfelds' surveys in 1930, several studies have pointed out the economical, psychological and relational consequences of the unemployment experience. But only few of them focus on the children's future. Nevertheless, the employment crisis in western countries since the mid-seventies has emphasized the issue of normative models in long-term unemployed families. This article is based on crossed interviews inside the families and tries to explain the logics of generational transmission in a context of transformation of the relation to work and employment. Two keys can help analyse the personal, domestic and social issues of this lasting crisis: a possible parental suffering and the opportunity for the child to build up interpersonal relationships based on mutual trust.

Key words: Unemployment, generational transmission, trust suffering 


\section{INTRODUCTION}

L'augmentation du chômage de longue durée des années 1970 et toute autre forme d'exclusion à très long terme du marché du travail incitent à s'interroger sur les repères normatifs transmis aux enfants par les parents sans emploi. Depuis les célèbres travaux de l'équipe de Lazarsfeld dans les années 30 à Marienthal (Lazarsfeld, Jahoda, Zeisel, 1981), nombreux sont les travaux de psychologues et de sociologues à avoir montré les effets dévastateurs du chômage sur le bien-être psychologique et physique (Hepworth, 1980; Gallie, Marsh et Vogler, 1994; Mc Kee-Ryan, Wanberg, et Kinicki, 2005) : tous ces auteurs soulignent la perte de repères, le repli sur soi, la honte et l'humiliation. La privation d'emploi est alors synonyme de manque, de carence et de déstructuration de l'individu.

Si ces questions demeurent centrales pour cerner la souffrance sociale, l'installation dans le chômage pose de nouvelles questions qui surgissent lorsque celui-ci s'installe durablement. Ainsi, des familles entières construisent leur existence loin des sphères toujours socialisatrices du travail salarié, loin des repères normatifs qui s’y réfèrent.

L’objectif de ce texte est d'apporter des éléments de réponse aux questions suivantes : à quelles transmissions générationnelles assiste-t-on lors d'une installation durable dans le chômage? Quelles sont les dynamiques identitaires, familiales et sociales qui peuvent se déployer dans un contexte de désintégration professionnelle? Quels sont les modèles normatifs qui sont transmis?

Ces questions sont très peu évoquées dans la littérature scientifique. Pourtant, elles risquent bien de s'imposer progressivement au cœur des réflexions politiques de demain. Comment en effet penser l'action publique d'une génération entière dont les parents ont été privés durablement d'une insertion professionnelle? Quel impact cette imputation sociale va-telle avoir sur une jeunesse en quête de sens?

\section{Conséquences du chômage parental sur les enfants}

Peu de recherches tentent de mesurer l'impact d'une situation de chômage sur la génération suivante. La problématique est récente et les difficultés méthodologiques très importantes. Comment en effet pouvoir isoler l'impact d'une expérience de chômage de longue durée sur la génération suivante?

Toutefois, certaines analyses récentes sont consacrées à ces questions. Certaines portent sur la carrière scolaire des enfants et le risque accru d'échec scolaire, d'autres sur le risque de transmission d'une situation de pauvreté entre les générations, d'autres enfin sur les vécus psychosociaux des enfants issus de familles privées d'insertion professionnelle. 
Un lien entre précarité professionnelle des parents et moins bons résultats scolaires obtenus par les enfants a été démontré (Duée, 2004; Bruniaux, Galtier, 2003; Dronkers, 1994; Christoffersen, 1994) : les enfants issus de familles pauvres réussissent moins bien que les autres. Leurs résultats sont inférieurs à l'école et sur le marché du travail, même si la mobilité intergénérationnelle est élevée. Mais ces auteurs mettent également en évidence les limites méthodologiques de leurs recherches en dénonçant l'insuffisance ou l'inadéquation de certaines données (trajectoires incomplètes, représentativité insuffisante des échantillons, variables de départ limitatives) ou de certaines méthodes employées (endogénéité du revenu, incertitudes liées à la relation entre revenu et résultats des enfants). Dronkers, aux Pays-Bas, met également en évidence une diminution des performances scolaires chez les enfants dont les parents subissent un chômage de longue durée, même si cette corrélation n’est pas toujours établie. La situation de chômage agirait comme un profond catalyseur sur des relations déjà existantes et ne ferait dès lors que renforcer des différences déjà présentes : plus exactement, le chômage a peu de répercussions (effet propre) sur les résultats scolaires de leurs enfants lorsque l'on contrôle les caractéristiques parentales les plus significatives. Mais il se combine avec les autres facteurs discriminants et les renforce (effet d'interaction). Les analyses danoises de Christoffersen confirment ces résultats : le risque de décrochage scolaire augmente parallèlement aux problèmes familiaux. Ce risque est fortement corrélé à plusieurs facteurs : l'isolement par rapport aux pairs, les difficultés de concentration à l'école et le changement fréquent d'établissement scolaire. Tous ces facteurs sont plus présents chez les enfants dont les parents subissent une situation de chômage de longue durée.

Aux États-Unis, une fois devenus adultes, près de la moitié des enfants nés de parents pauvres n'échappent pas à la pauvreté (Corak, 2004). Pour mesurer la mobilité intergénérationnelle des gains sur le marché du travail, Corak reprend l'ensemble des études disponibles (fondées sur un modèle empirique simple utilisant le revenu permanent et un indice pour la génération). Au départ de cette équation, on cherche à déterminer la fraction de revenu qui se transmet. Il s'agit en quelque sorte de l'élasticité intergénérationnelle des revenus. Par sa méthode critique, il met rapidement en lumière des divergences internationales significatives, en termes de résultats et en matière de politiques sociales et plus précisément en termes de politiques d'investissements et émet l'hypothèse que, si l'argent a une emprise conséquente sur le devenir à long terme des enfants, d'autres facteurs existent, d'une portée aussi significative : les différences observées entre pays résultent également du haut niveau de salaire des plus qualifiés et des opportunités offertes à leurs enfants d’acquérir des compétences et diplômes.

Les enfants, issus de familles dont aucun des parents n’a pu être à l'emploi régulièrement, diffèrent-ils des autres enfants en ce qui concerne la santé et le bien-être? Plusieurs recherches tendent effectivement à démontrer que le chômage peut entraîner une détresse mentale (Reinhardt Perdersen et Madsen, 2002; Dronkers, 1994; Christoffersen, 1994). Cette perte d'emploi induit une chute du statut social, une rupture des rôles, une perte d'estime de soi et l'accroissement des difficultés financières. Or, ces changements qui affectent la santé mentale parentale rejaillissent sur les enfants (hypersensibles à l'état émotionnel des parents). Reinhardt 
Perdersen et Madsen observent que des groupes se marginalisent par leur non-participation au marché de l'emploi. Les recherches de Dronkers et celles de Christoffersen rejoignent les résultats de Reinhardt Perdersen et Madsen et montrent combien les effets du chômage de longue durée subi par les parents se répercutent sur la santé mentale des enfants et cela à plusieurs niveaux. Pour Christoffersen, l'expérience du chômage engendre chez certains parents une perte de statut social, une déstructuration des rôles sociaux, une perte de l'estime de soi et une diminution des revenus du ménage. Toutes ces conséquences peuvent également engendrer des effets indirects sur la vie des enfants. En effet, l'auteur constate une diminution des activités de sociabilité chez les enfants et une tendance à l'isolement social : les loisirs se concentrent à la maison et les contacts avec l'extérieur sont moins fréquents. Les enfants hésitent à amener leurs compagnons de jeu à la maison, soit à cause d'un refus parental, soit à cause de la honte provoquée par la situation des parents. L'estime de soi des enfants est également menacée à long terme. Plus fragiles, ils souffrent d'un manque de confiance en eux, de problèmes d'insomnie, de cauchemars fréquents ainsi que de crises d'anxiété. Mais ces effets dépendent de l'ambiance familiale et de leur capacité à fonder à leur tour un couple stable. En effet, toutes ces conséquences sont médiatisées par toute une série de facteurs issus de leur enfance et du degré de crispation autour de la situation de chômage, mais également de facteurs postérieurs à l'enfance, tels que le fait de trouver un emploi stable ou un(e) conjoint(e) stable. Ils parviennent dès lors à dépasser les troubles de leur enfance au profit d'une stabilité professionnelle ou conjugale.

Ces recherches démontrent assez bien l'impact du chômage parental sur le vécu et l'avenir de leurs enfants. Les conséquences sont multiples et diverses. Mais elles mettent également en évidence la complexité de la relation et l'aspect multidimensionnel d'une telle problématique. On ne peut en effet considérer l'impact du chômage sur la génération suivante sans distinguer les différents agents qui viennent médiatiser cette relation. Comment saisir les différents paramètres qui interfèrent entre eux et qui soit enferment le jeune dans une spirale de reproduction du social, soit lui permettent de dépasser ce risque et cet enfermement?

\section{Méthodologie}

D’octobre 2000 à mars 2004, une recherche ${ }^{1}$ a été menée en Belgique sur la question de la transmission de modèles normatifs chez les chômeurs de longue durée ${ }^{2}$. L'objectif était d'identifier les devenirs professionnels des enfants issus d'un contexte de chômage parental et de déterminer les mécanismes de (re)production de modèles normatifs sur deux générations. Il s'agissait moins de montrer combien le risque du chômage pouvait se transmettre d'une génération à l'autre que d'identifier les modèles normatifs transmis, notamment en matière de

1. Recherche intitulée «Chômage et transmissions générationnelles », financée par le Fonds National de la Recherche scientifique (2000-2004).

2. Notons que la Belgique se caractérise par un système de protection sociale où il n'existe pas de fin de droit en matière de chômage. On peut donc rencontrer des expériences de chômage de plus de 15 ans, voire 20 ans. 
rapport à l'emploi : comment saisir les logiques de transmission générationnelle ${ }^{3}$ qui parviennent à déporter sur la génération suivante l'expérience du chômage parental? Ces logiques de transmission sont à rechercher dans le jeu incessant de travail de (re)construction identitaire des différents protagonistes où s’entrechoquent le souci d'être soi, les dynamiques familiales et les impératifs normatifs du social.

Le dispositif de recherche mis en place s'articule autour de la réalisation et de l'analyse d'entretiens croisés. Il s'agit de confronter, au sein d'un même espace d'analyse, les récits obtenus par interviews de différents personnages issus d'un même cadre de vie. Pour chaque famille, nous avons systématiquement interrogé le parent référent au chômage ainsi qu'au moins un enfant, âgé de 16 à 25 ans, vivant toujours chez ses parents, sorti ou pas du circuit de la scolarisation. Tous les entretiens ont été réalisés sans la présence des autres membres de la famille. Nous avons ainsi réalisé 42 entretiens ${ }^{4}$ dans 18 familles différentes. Le nombre d’entretiens réalisés a été fixé par les impératifs de recherche, par saturation des données.

Il nous paraissait en effet important de pouvoir appréhender les vécus individuels ainsi que les interactions créées entre les membres de la famille. C'est dans la gestion de la quotidienneté que se transmettent les modèles normatifs, au cœur du jeu entre ce qui est dit et ce qui est tu. La capacité de narration des différents protagonistes sur leur propre parcours, mais aussi sur les représentations qu'ils se font de l'autre permet de reconstituer un espace de rencontre analytique qui n'appartient ni à l'un, ni à l'autre, mais qui se situe au cœur même d'un espace d'interactions formé entre eux.

C’est déjà ce qu’avait fait l’anthropologue américain O. Lewis, dans son célèbre ouvrage «Les enfants de Sanchez », en interrogeant longuement les membres d'une famille vivant dans un bidonville de Mexico (Lewis, 1986). Les récits du père, des sœurs et des frères lui avaient permis d'appréhender sous des angles différents des événements familiaux vécus parfois de manière radicalement opposée. Cette mosaïque de récits permet au lecteur de saisir, à travers les subjectivités de chacun, ce que c'est que de vivre dans la pauvreté lorsque l'on est issu d'une famille récemment arrivée de la campagne et obligée d'habiter le bidonville. La diversité permet d'approcher la complexité d'une culture familiale nécessairement influencée par des conditions de vie spécifiques: ces discours (...) apportent des éléments indispensables à la saisie des

3. On ne peut appréhender ces questions sans s’interroger un moment sur les présupposés épistémologiques qu'ils génèrent. Comme le souligne toujours Lahire, le concept de transmission est ambigu parce qu'il souligne une forme d'unilatéralité de la relation : le destinataire ne faisant finalement que recevoir sans rendre et sans construire. L'héritage serait alors comme un testament que l'on ne peut qu'accepter sans le modifier. Aucun travail d'appropriation ne serait dans ce cas possible. Le terme d'échange générationnel permettrait de rétablir l'équilibre de la relation, mais occulterait quelque peu la force du pouvoir de la socialisation. De fait, le déséquilibre existe entre ce qui se transmet d'une génération à l'autre. Nous avons finalement opté pour le concept de « logiques de transmission générationnelle » qui tente de concilier les deux remarques.

4. Entretiens semi-structurés dont la grille d’entretien contenait les thématiques suivantes : trajectoire scolaire, trajectoire professionnelle (pour le parent), expérience du chômage et de son vécu, situation et vécu familiaux, structuration du temps et de l'espace, représentations de l'avenir, réseau social et sociabilité. 
Revue Service social

dynamiques familiales et aux interactions d'un membre du groupe familial. Apparaissent dès lors les intérêts communs, mais aussi les objectifs et les priorités individuels (Delcroix, 1995, p. 62).

L’intérêt de la démarche repose donc à la fois sur la multiplication des vécus individuels et sur la possibilité de croisement des subjectivités.

D’un point de vue méthodologique, le déploiement d'un tel dispositif méthodologique permet de travailler les questions de validité de l'approche qualitative sous l'angle de la triangulation (Tashakkori, Teddie, 1998). Celle-ci repose en effet sur la multiplication d'angles d'entrée complémentaires qui se croisent au sein d'un même dispositif de recherche. Elle peut être envisagée sous différents aspects : triangulation des données (l'utilisation de sources de données différentes), triangulation des chercheurs (utilisation de différents chercheurs qui confrontent leurs analyses), triangulation des théories (l'utilisation de perspectives théoriques multiples pour l'interprétation des résultats d'une enquête), triangulation méthodologique (l'utilisation de techniques d'enquêtes différentes). La multiplication des récits narratifs permet de croiser les dires et d'ainsi mieux cerner les espaces de rencontre.

D'un point de vue théorique, l'analyse se fait plus fine et permet de mieux confronter les hypothèses émises. Les logiques de transmission apparaissent plus clairement parce que les témoignages se font face et peuvent être interprétés l'un par rapport à l'autre dans un même espace analytique.

Pour tenter de comprendre cet espace analytique, nous prendrons un exemple tiré des entretiens réalisés avec les familles. Ils montrent l’intérêt de la démarche méthodologique choisie en mettant en évidence les différences d'interprétation possible entre l'analyse d'un seul récit narratif et l'analyse croisée rendue possible par la multiplication des récits. Les récits s'entrechoquent et laissent apparaître des structures communicationnelles qui, soit se font écho, soit s'opposent, mais qui s’ignorent la plupart du temps; un peu comme si elles s'élaboraient en parallèle, c'est-à-dire l'une par rapport à l'autre, mais sans qu'il existe de point de rencontre entre elles. Comment puis-je comprendre cet Autre qui m'est si proche et pourtant si lointain?

\section{Le vilain petit canard}

Christine a 44 ans, elle vit seule avec sa fille Jessica, âgée de 19 ans. Elle est au chômage depuis de nombreuses années. L'entretien n'est pas facile, la relation difficile à établir, la confiance difficile à nouer. Mais peu à peu, s’instaure un climat plus détendu, plus serein qui permet un véritable échange. Elle parvient à se livrer, à confier les démons de son passé.

L’expérience du chômage est douloureuse, elle est vécue comme une contrainte supplémentaire dans la vie de Christine: perte des repères, repli sur soi, carences financières... Mais plus encore, la vie de Christine est parsemée d'embûches, de drames familiaux et de problèmes de santé importants. Issue d'une famille nombreuse, comptant six frères et sœurs, elle a subi 
pendant son enfance le poids du malheur et des crises familiales : précarité professionnelle, père alcoolique, violence familiale... On sent à travers la parole toute la souffrance encore enfouie. L'entretien sert finalement de soupape permettant d'évacuer un trop-plein. Elle nous confiera l'indicible, celui d'un père qui abuse de ses filles au fur et à mesure que survient leur puberté. Le poids du silence, l'angoisse qui ronge et les sentiments de culpabilité et de souillure qui l’envahissent. Elle garde à tout jamais les séquelles profondes de cette période.

Au fil du discours, on comprend en effet combien cette enfance terrible a guidé ses choix futurs, mais aussi combien il est difficile d'échapper au malheur. Pour fuir l'étouffement familial, elle se réfugie dans le mariage. Mais là encore, la violence conjugale est au rendez-vous. De ce mariage naissent deux filles, Alisson, mariée et mère de trois enfants, et Jessica, 19 ans, enceinte d'un petit garçon. Elle nous dira combien ce sont ses filles qui lui ont permis de continuer à vivre et combien elle donnerait tout pour qu'elles soient bien.

Il y a quelques années, Christine a voulu revenir sur son passé. Elle est allée voir sa mère et ses sœurs, elles aussi victimes, pour enfin dire sa souffrance. Mais le poids du silence a repris le dessus : à quoi bon remuer ainsi un passé vieux de plus de vingt ans? Mais Christine n’a pas voulu se taire une fois de plus, elle a déposé plainte contre son père et une enquête a été ouverte. Toute la famille a fait bloc... contre elle en niant la véracité des faits. Cette négation même de l'existence de sa souffrance a profondément marqué Christine au point de provoquer chez elle un besoin de s'interroger sur sa position familiale. Elle nous dira à ce sujet: "chez moi, j'ai toujours été le vilain petit canard, celui qui ne fait pas comme il devrait faire, celui qui n'est pas comme les autres et que personne n'aime vraiment. J'ai toujours été à part et encore maintenant, il y a des réunions de famille, pour la Noël par exemple, et bien, moi, je ne suis jamais invitée. Je suis de trop. J'ai toujours senti que j'étais de trop, celle qui ne suit pas les autres..."

L’important dans ce récit n'est pas que Christine soit ou ne soit pas ce vilain petit canard, mais bien qu'elle s'en serve dans son récit narratif et la place qu’occupe cette métaphore dans la reconstruction du récit de sa vie.

Jessica a 19 ans, elle est la sœur cadette d'Alisson. Elle vit avec sa mère dans un appartement du centre-ville et elle attend son fils avec impatience au moment de l'entretien. Son ami vient de manière sporadique et Jessica ne supporte pas son comportement indécis quant à sa future paternité. Elle voudrait un engagement plus formel et cela d'autant plus, nous dira-t-elle, que lui travaille et qu'elle est au chômage. Au fil d'un discours très débridé, elle nous dira combien elle craint qu'il n'assume pas son rôle de père, un peu comme le sien avait laissé tomber sa mère. À nouveau, le poids de la reproduction générationnelle pèse sur le devenir familial. Elle nous dira combien le départ de ce père a à la fois soulagé l'atmosphère familiale et crispé les relations entre la mère et ses filles. Jessica nous dira combien elle a souffert de ce départ parce qu'elle était proche de son père, mais surtout parce que sa mère a à la fois rejeté ce mari violent et cette fille trop proche de lui. Elle nous dira à ce propos : "Elle a toujours préféré ma sœur, c'était toujours elle qui faisait bien, qui rangeait sa chambre, qui était gentille... Moi, j'étais en trop, celle qui rappelait mon père à ma mère. Sa façon de me regarder, son comportement avec moi... 
J'étais pas désirée, j'étais comme un canard noir chez les canards blancs...»" Ces deux récits nous montrent la complexité des relations entre Christine et Jessica. Christine qui insiste sur l'importance de ses deux filles dans son cheminement identitaire et Jessica qui crie son mal-être dans la relation qu'elle entretient avec sa mère. Les conflits entre la mère et la fille sont d'ailleurs légion et ne font que renforcer les positions de chacune.

L’analyse de ces entretiens croisés fait apparaître toute l'ambiguïté de cette relation. L’important n’est évidemment pas de dire qui a raison, mais bien d'acter les différences de perceptions entre la mère et la fille. La compréhension de la dynamique familiale s'enrichit du croisement des récits narratifs.

De la même manière, la similitude des métaphores employées montre la force des mécanismes de transmission. Alors que Christine crie sa souffrance dans la négation de sa place familiale, Jessica lui fait écho en criant cette même souffrance, mais sans qu'elles ne puissent s'entendre mutuellement...

\section{Résultats}

L'analyse des entretiens croisés permet la construction d'une typologie des cadres de la transmission au sein de l'espace analytique. Cette typologie est construite à partir de deux clés interprétatives. Celles-ci permettent d'appréhender les logiques de transmission à la fois par rapport aux jeux identitaires des différents protagonistes et par rapport aux modèles normatifs mis en question.

\section{Deux clés interprétatives}

Une première clé de compréhension des logiques de transmission est à rechercher chez le parent chômeur et plus précisément dans sa manière d'appréhender sa situation de chômage.

Il peut en effet vivre son expérience du chômage comme un véritable drame social : la privation d'emploi s'accompagne alors d'un nœud de souffrance qu'il ne peut surmonter. L'angoisse du lendemain, l'incertitude professionnelle, la honte et l'humiliation, l'isolement social sont autant d'éléments de déstructuration des repères identitaires. Cet état a déjà fait l'objet de multiples études qui mettent toutes en évidence la profonde détresse psychologique et sociale qui accompagne un chômage de longue durée ou de très longue durée. L'individu perd ses repères sans pouvoir se reconstruire et finit par perdre espoir d'un jour pouvoir regagner le marché de l'emploi.

Pour d'autres, au contraire, l’expérience du chômage est un moment de crise qui, certes, déstructure le quotidien, mais qui ne peut être dépassé par un travail identitaire de reconstruction de soi et de son rapport aux autres. La souffrance ne s'installe pas durablement, même si le chômeur connaît des moments de blues. 
La deuxième clé interprétative doit être recherchée dans le concept de confiance. La confiance, pour A. Giddens, peut être définie comme un sentiment de sécurité justifié par la fiabilité d'une personne ou d'un système, dans un cadre circonstanciel donné, et cette sécurité exprime une foi dans la probité ou l'amour d'autrui, ou dans la validité de principes abstraits (Giddens, 1994, p. 41). Plus précisément, l'élaboration d'un espace d'échange construit sur un mode égalitaire de partage et d'écoute, propice à l'émergence de rapports de confiance interpersonnels, offre à l'individu une opportunité de dévoilement de soi, de son histoire, de son vécu. Pour reprendre P. Ricoeur : compter sur quelqu'un c'est à la fois faire fond sur la stabilité d'un caractère et s'attendre que l'autre tienne parole, quels que soient les changements susceptibles d'affecter les dispositions durables à quoi il se laisse reconnaître (Ricoeur, 1990, p. 176). Ce « être-là pour quelqu'un » permet alors d'ouvrir un espace de réflexivité par rapport à sa situation, à son existence et à son expérience. Dans ce sens, les rapports de confiance doivent être compris comme une relation, un éprouvé et un construit qui nécessitent le regard bienveillant de l'Autre et qui doivent sans cesse se donner à voir par des témoignages symboliques afin de réassurer de leur existence.

Le cadre familial constitue un ancrage privilégié de ces rapports de confiance. Il peut générer un climat propice à l'instauration d'un espace de rencontre et d'écoute. Dans ce sens, le sentiment de continuité de la relation fonctionne comme un préalable au dévoilement : je peux dire ce que je ressens parce que je sais que tu étais là, que tu es là et que tu seras là. Le caractère durable de la relation, fondé ou non, permet à l'individu d'établir des rapports de confiance basés sur un face-à-face expérientiel, où les réminiscences de l'action commune constituent un substrat nécessaire au dévoilement de soi, et permet également de se projeter dans l'avenir. Passé, présent et avenir se conjuguent alors dans un même espace expérientiel symbolique et imaginaire nécessaire à la persistance des rapports de confiance.

Le déploiement de l'identité narrative présuppose également le partage de référents normatifs communs, voire même d'une certaine vision du monde. Plus précisément, l'existence d'un espace normatif commun permet de cadrer l'interaction et de mettre en place les conditions nécessaires à l'instauration de rapports de confiance propices à ce déploiement. Sans leur présence, l’individu est condamné à se replier sur son intimité.

De la même manière, l'impossibilité d'instaurer des rapports de confiance peut provenir d'un sentiment de trahison où le dévoilement a débordé du cadre de l'intimité où il doit rester confiné. Cette trahison rompt les rapports de confiance déjà instaurés, mais rend également extrêmement difficile la mise en place d'un nouveau système de confiance avec d'autres partenaires, un peu comme si la rupture d'une relation privilégiée ne pouvait engendrer qu'un sentiment de méfiance difficile à dépasser. L'émergence de relations basées sur le reproche et l'incompréhension dénoue toute possibilité d'établissement de rapports de confiance, prive par là l'individu d'un travail sur son identité narrative. 
Si la famille constitue un espace privilégié de construction de rapports de confiance, elle n’en est pas la seule génératrice. D’autres espaces peuvent surgir et s’imposer progressivement. Les sphères de sociabilité peuvent en effet également être au cœur du déploiement des dispositifs de confiance interpersonnelle. Mais les conditions d'émergence suivent les mêmes règles et imposent les mêmes exigences.

Exigence de fiabilité tout d'abord : l'individu doit être convaincu que le partenaire ne trahira pas la relation établie.

Exigence d'un espace de liberté ensuite: le dévoilement de soi ne peut s'élaborer à l’intérieur d'un rapport de pouvoir ou de contrôle social.

Exigence temporelle encore : le temps doit pouvoir s’installer, dénoué de toute contrainte de résultats à atteindre impérativement et immédiatement.

\section{Typologie des modèles de transmission}

Les deux clés de lecture présentées précédemment nous aident à mieux appréhender les logiques de transmission et les modèles transmis. Elles se combinent en effet au sein d'un même espace transactionnel et composent des figures différentes que l'on peut typologiser.

\section{Absence de souffrance parentale et reproduction des modèles normatifs}

La première figure identifiée s'inscrit dans une logique de reproduction du modèle parental. L'expérience du chômage ne suscite pas chez le parent qui la subit une souffrance qu'il ne peut surmonter. Il parvient à gérer son écartement du marché de l'emploi grâce aux soutiens dont il dispose. Ces soutiens sont de nature différente, mais concourent tous à pouvoir relativiser la situation de crise qui accable le chômeur.

Les ressources sont tout d'abord d'ordre matériel. L'écartement prématuré du marché de l'emploi déstabilise l'apport financier du ménage, mais sans créer de véritable gouffre financier. Soit l'individu dispose de réserves confortables lui permettant d'appréhender sereinement le lendemain, soit la période de chômage coïncide avec la fin du remboursement de son crédit hypothécaire et la mensualité compense la perte de salaire. Le conjoint peut également décider d'augmenter son temps de travail temporairement. Des stratégies familiales sont ainsi déployées de manière à dépasser la crise financière. Celles-ci sont d'autant plus faciles à mettre en œuvre que la période de chômage survient après 50 ans. Des politiques sociales existent en effet en Belgique pour les chômeurs âgés qui leur permettent de bénéficier de droits sociaux plus avantageux que les autres demandeurs d'emploi.

Les ressources sont aussi relationnelles et familiales. Le vécu du chômage est également influencé par le soutien apporté par les proches. Le conjoint et les enfants établissent un véritable dialogue avec le chômeur qui l'aide à surmonter l'épreuve. C'est dans l'espace transactionnel que 
se construisent les stratégies identitaires lui permettant de faire face et de progressivement accepter sa perte d'emploi. Le cadre familial devient alors un refuge sur lequel le chômeur peut compter lorsque la pression devient trop difficile à gérer, dans les périodes de doute ou de découragement.

Les ressources enfin sont aussi individuelles. Elles témoignent d'une capacité de l'individu à se prendre en main, à ne pas s'effondrer psychiquement et mentalement. Plus ses ressources personnelles sont présentes, plus le chômeur parviendra à dépasser le moment de crise que suscite inévitablement la perte d'emploi. Il existe bien entendu des différences interindividuelles importantes. Sans entrer dans des considérations psychologiques, ces ressources mobilisables dépendent des parcours individuels : au plus l'individu a déjà dû faire face à de nombreuses épreuves de vie, au plus il éprouvera des difficultés à accepter et à dépasser cette dernière crise, surtout lorsqu'aucun soutien relationnel n'est venu précédemment.

Toutes ces ressources ne peuvent à elles seules expliquer la relative absence de souffrance liée à l'expérience du chômage. Il faut en effet également nous interroger sur l'activité professionnelle et ce qu'elle représente pour le chômeur, au cœur des modèles culturels dominants. L'activité professionnelle peut demeurer une valeur fondamentale dans la vie de l'individu, mais il est soumis à des impératifs normatifs construits autour de l'épanouissement personnel et la réalisation de soi. Dans ce sens, l'activité professionnelle devient un lieu d'épanouissement et de réalisation de soi, mais cette aspiration à l'épanouissement peut également s’incarner à l'extérieur du monde professionnel. On assiste alors à une vision polycentrée de l'existence où l'investissement identitaire peut aussi bien se nouer à l'intérieur du travail qu’à l’extérieur de celui-ci.

Dans ces conditions, le poids de l'expérience du chômage n'est pas prégnant à la génération suivante. Tout se passe comme si les questions de gestion identitaire restaient enfermées chez le parent chômeur. Elles ne transparaissent pas à la génération suivante. Le jeune n'est pas atteint dans son identité : il intègre l'expérience parentale comme un aléa de la vie professionnelle qui peut survenir à n'importe qui et à n'importe quel moment. Il parvient donc à mettre à distance l'expérience du chômage parental et à la contextualiser dans un environnement économique perturbé et difficile. Elle devient une épreuve qui n'affecte pas les liens de confiance qui se nouent entre les membres de la famille. Elle n'est pas absente du discours, mais elle est intégrée à l'intérieur d'une conception relativisée des risques de chômage. Il peut dès lors continuer son propre cheminement identitaire sans être affecté au plus profond de son être.

On se trouve dans un modèle de reproduction du modèle parental parce que le jeune adhère lui aussi au modèle de l'épanouissement personnel et de la polycentration. L'activité professionnelle a certes une importance dans la projection existentielle, mais doit être relativisée en fonction d'autres sphères tout aussi importantes à ses yeux. L'essentiel est de s'épanouir, de se réaliser à l'intérieur du monde professionnel ou en dehors. Les lieux d'investissement sont 
multiples et variés, mais ils permettent de ne pas se centrer uniquement sur le travail comme condition nécessaire et suffisante à l'épanouissement personnel. transmission ${ }^{5}$.

L’exemple suivant permet de mieux appréhender cette première figure des cadres de la

\section{Le prix de la passion}

Dominique a 55 ans, divorcé depuis une dizaine d'années et père de trois enfants : Louis (21 ans), Daphné (19 ans) et Antoine (15 ans). Il est photographe de profession et décorateur d'intérieurs. Son activité professionnelle connaît des hauts et des bas en fonction des contrats qui se présentent à lui. Dominique est conscient de cette précarité, mais l'assume : " c'est le prix à payer pour exercer sa passion. Et puis, à l'heure actuelle, plus personne n'est véritablement à l'abri ». Le travail est avant tout une passion et son statut lui procure une certaine forme de liberté, de disponibilité et de flexibilité. L’argent ne fait pas le bonheur et il préfère sacrifier le surplus plutôt que le bien-être.

Dominique a une vision précise de son rôle paternel et de l'éducation à donner à ses enfants. Tout se joue dans la transparence et dans la confiance : "je ne cache rien aux enfants, je leur laisse choisir leur voie, je leur fais confiance. L'important, c'est de les responsabiliser et de leur laisser leur autonomie. Je reste en contact, je dialogue beaucoup et j'aime prendre du temps avec eux. Mon activité professionnelle me laisse des moments à partager avec eux... »

Louis a 21 ans et vient de terminer une licence de philosophie à l'université. Sa chambre est remplie de livres de philosophie et il nous raconte que l'attrait pour la philo provient de la bibliothèque paternelle. Plus exactement, adolescent, Louis devait traverser le bureau de son père pour se rendre dans sa chambre. Ce bureau contenait la bibliothèque de son père et c'est tout naturellement que Louis y a jeté un coup d'œil par-ci, par-là, jusqu'au moment où il est tombé par hasard sur un manuel de philo qui datait du bac de son père. Cette banale découverte va transformer sa vie au point de faire naître chez lui une véritable passion. Louis avait trouvé sa voie.

Cette découverte va également lui permettre de se tracer une ligne de conduite à suivre dans sa scolarité : il prend conscience de l'importance du parcours scolaire qu'il gère en fonction de son objectif final. Cette quête lui procure donc un cadrage de son existence.

À ses yeux, on peut établir un parallèle entre la philo et le métier artistique de son père. Ce qui compte c'est de pouvoir se réaliser dans son métier et de pouvoir s’épanouir en exerçant sa passion. Il ne peut imaginer un travail où il serait « prisonnier » d'un emploi du temps précis, de contraintes organisationnelles fortes. Il rêve d'un engagement à l'université où il pourrait réaliser une thèse de doctorat et «être payé pour lire ». Il entreprend d'ailleurs des démarches dans ce sens.

5. Les exemples permettent de mieux comprendre la construction de l'idéal type, mais ils ne contiennent pas, de manière individuelle, l’ensemble des caractéristiques de cet idéal type. 
Il est assez lucide sur la difficulté de réaliser ce défi, mais se dit prêt à aller au bout de son rêve : « il sera toujours bon de voir, si vraiment cela ne marche pas ».

Ces deux récits nous montrent combien le modèle paternel s'est transmis à Louis qui préfère s’investir dans une passion, quitte à ne pas choisir la sécurité d'emploi. On peut établir un véritable parallèle entre les termes utilisés par Dominique pour décrire son activité professionnelle et les termes de Louis lorsqu'il parle de son désir de continuer à travailler la philo. Les mécanismes de la transmission se laissent appréhender par le récit croisé. Ce n’est pas tant l'objet qui fait transmission que les logiques véhiculées. Le hasard n'est pas non plus absent du processus puisque Louis nous montre combien la rencontre avec le livre de philo a constitué un véritable filet de cadrage nécessaire pour pouvoir s’élaborer un projet de vie.

\section{Souffrance parentale et construction de rapports de confiance}

Dans ce second modèle, l'expérience du chômage est dramatique. La perte d'emploi constitue un choc psychologique et relationnel dont l'individu ne peut se défaire. On retrouve ici l'ensemble des conséquences décrites par la littérature sur le vécu du chômage : un sentiment de honte et d'humiliation, une perte des repères spatiaux et temporels, un isolement social... On assiste à un véritable effondrement identitaire du chômeur. Il ne parvient pas à surmonter la période de crise, il y reste englué. La souffrance est omniprésente et transpire véritablement de l'individu.

Cette souffrance s'explique bien entendu par le choc né de la perte d'emploi, mais également par la place symbolique qu'occupe l'activité professionnelle dans son schéma identitaire. Elle structure véritablement l'individu, lui confère un statut social, mais plus encore; elle lui donne une raison d'exister. Au plus l'individu est centré sur son activité professionnelle, au plus la perte de celle-ci est difficile à assumer. C'est alors tout l'investissement de toute une vie qui s'écroule, laissant un vide difficile à combler du jour au lendemain. Nier son importance reviendrait à trahir son passé et dès lors à se trahir. Continuer à croire en sa centralité reviendrait à reconnaître son échec. Seule l'espérance de regagner le marché de l'emploi permet au chômeur de se reconstruire autour de ce projet. Mais au fil des mois, l'espoir s'estompe et seul demeure la souffrance, et cela d'autant plus que les ressources sont peu nombreuses ou inexistantes. Elles ne peuvent aider le chômeur à se reconstruire.

Il est souvent pris dans un jeu financier dont il ne peut que sortir perdant. La perte de salaire n'est pas compensée, les difficultés financières s'accumulent progressivement et établissent une véritable pression sur un éventuel retour à l'emploi. Le temps joue contre le chômeur. Surviennent alors les situations de surendettement, de crédits multiples, sensés pallier provisoirement la diminution des apports financiers du ménage. Le conjoint ne parvient pas à combler le trou et ce trou se transforme en gouffre.

C'est également le temps des reproches qui accablent plus encore parce qu'ils enferment dans une logique de responsabilité et de culpabilité. Ils indiquent implicitement ou explicitement 
les torts du chômeur en faisant reposer sur ses épaules la situation que toute la famille supporte : raisons du licenciement, difficultés à retrouver un emploi... Le chômeur finit par perdre toute légitimité parentale. Socialement déstructuré et affaibli, il ne parvient plus à assumer son rôle parental et sort perdant du rapport de force symbolique qui doit présider à toute forme d'éducation. Il finit par ne plus être crédible aux yeux des enfants et ne peut plus constituer un modèle à suivre.

Le jeune ne peut prendre appui sur un modèle parental qui s'effondre progressivement. Mais il trouve des repères ailleurs qui vont l'aider à se construire. Il peut s'agir d'un autre membre de la famille ou même d'une connaissance. Le schéma d'identification demeure basé sur l'établissement de rapports de confiance interpersonnels qui structurent les logiques de transmission. En l'absence d'un modèle parental fort, le jeune trouve chez un tiers un modèle d'identification lui permettant de se construire une identité forte. Sa conception de l'activité professionnelle sera dès lors fortement influencée par les liens de confiance interpersonnelle qu'il aura pu progressivement nouer. Soit il adoptera une conception unicentrée de l'activité professionnelle qui structurera l'ensemble de son schéma identitaire, soit il pourra l'élargir en fonction d'une adhésion à un modèle polycentré basé davantage sur un épanouissement personnel.

L'exemple suivant permet à nouveau d’illustrer la seconde figure.

\section{La rupture}

Marie-Paule est divorcée et mère de deux enfants. Julie, 23 ans, Nadine, 20 ans. Marie-Paule est au chômage depuis plus de trois ans. Son mari et elle ont toujours alterné les emplois précaires avec des périodes de chômage. Elle a tendance à se renfermer sur elle et à s'isoler progressivement de son réseau social. Elle tente de rationaliser sa situation en dédramatisant, mais son discours sonne faux par moment : "l'activité professionnelle n'est pas le seul élément structurant dans une vie ». Elle insiste sur la qualité de vie et la mise à distance de l'emploi : « $A$ quoi bon faire une carrière, grimper les échelons... pour moi, la vie, c'est bien autre chose ». Mais on sent que ces propos témoignent davantage de la justification de sa situation que d'une croyance profonde : elle alterne notamment les passages nostalgiques, "Bon d'accord, avant, on pouvait partir en vacances l'été et puis s'offrir des petits moments sympa, mais maintenant c'est plus difficile ». À d'autres moments encore: "j'ai bien essayé de retrouver un boulot plus stable, mais à mon âge, on est déjà vieux ».

Julie termine actuellement un graduat en comptabilité, mais travaille déjà comme comptable dans un hôtel de la capitale. Les relations avec sa mère sont difficiles. Elle lui reproche de se complaire dans sa situation de chômage et ne supporte pas que sa mère " profite du système et de l'argent des autres ». Mais au-delà du reproche de cette situation, c'est tout le modèle véhiculé par sa mère qu'elle rejette violemment. «La vie, c'est quand même pour bosser, pour évoluer. J'ai eu un drôle d'exemple avec mes parents, parce que normalement tu as un des deux parents qui bosse normalement. Et moi, aucun des deux n'a bossé normalement ». Son modèle s’oppose 
de manière caricaturale à celui de ses parents. Elle prône l'investissement professionnel, l'évolution de carrière et surtout le gain financier. Pour elle, la réussite passe automatiquement par l'importance du salaire. "L'argent, tout passe par là. Je n'étais pas comme les autres parce qu'il fallait toujours se priver. Ma fierté et ma reconnaissance personnelle passent par le matériel... » La construction identitaire passe par la possibilité de consommer : j’existe par mes capacités à consommer.

Mais la rupture du modèle familial révèle un autre aspect de la personnalité de Julie : son besoin de conformité et de sécurité. Elle rêve d'une vie qui lui permettrait de se fondre dans un moule, de rejoindre ce qu'elle considère comme la norme et de "faire enfin comme les autres ». Le modèle prôné par ses parents était générateur d’angoisse chez Julie : la peur du lendemain, de la précarité et de l'incertitude ont pesé jusque dans ses choix d'étude. «Un jour, ma prof de math m'a dit que je pourrais faire des études supérieures. Elle m'a guidée dans les choix, elle m'a écoutée et vraiment conseillée. Ça été important pour moi à un moment de ma vie où je ne savais plus vraiment où j'allais ». Elle nous dira à ce propos qu'elle a choisi la comptabilité pour les débouchés qu'un tel diplôme lui procurerait et non pas par goût du métier. L’important, c'est de trouver un emploi sûr qui rapporte suffisamment d'argent pour assouvir son besoin de consommer.

\section{Souffrance parentale indépassable et production de l'échec}

Le troisième modèle est également celui de la souffrance parentale. Elle est présente dans les discours du chômeur, elle transpire également au sein de la famille. C'est le même manque de ressources matérielles, psychologiques, relationnelles qui accablent le foyer parental: l'expérience du chômage déstructure profondément les relations familiales. Naît alors un climat tendu, presque macabre, qui rejaillit sur la génération suivante.

Contrairement au modèle précédent, le jeune n’a pu nouer de rapports de confiance interpersonnelle sur lesquels il pourrait prendre appui pour se construire, soit parce qu'il n'a pu les trouver, soit parce qu’il a été trahi. Sans cadre structuré et structurant, il s'enferme progressivement dans un univers noir de l'échec. Décrochage scolaire, instabilité relationnelle et professionnelle, il est pris dans une véritable spirale de l'échec et cela d'autant plus que les conditions d'accès au marché de l'emploi sont difficiles. Il continue à adhérer à un modèle où l’emploi est prégnant, mais inatteignable.

Il ne peut concevoir l'avenir à long terme : seul le quotidien est présent dans un discours peu cohérent où se mêlent réalités et affabulations. Il a, à la fois une capacité réflexive qui lui fait comprendre le drame de sa situation, mais aussi une capacité imaginative qui l'emmène dans des mondes fabuleux où il est enfin quelqu'un de bien.

Parfois, la seule échappatoire est le monde de la rue, de la débrouille et de l’illégalité. Ce monde parallèle où les règles existent et où il peut trouver le cadrage nécessaire à sa vie psychique (Jamoulle, 2002). Il peut procurer des ressources matérielles, mais aussi une identité 
sociale et une position sociale. Les conduites à risque (dépendances, violences, petits trafics...) se multiplient alors dans la clandestinité sans que les familles sachent véritablement lutter contre.

Le dernier exemple illustre cette transmission de l'échec.

\section{L’impasse}

Louis est au chômage depuis plus de quinze ans. Ancien ouvrier, employé dans le secteur sidérurgique, il a été la victime des restructurations successives des bassins industriels. Il habite une région où le taux de chômage dépasse les 20 \% de la population active. Sa femme n’a jamais travaillé et leurs ressources se limitent aux allocations de chômage qu'il perçoit en tant que chef de ménage. Ils ont un fils, Stéphane, 19 ans, en décrochage scolaire. La vie de Louis est vide, il est totalement apathique et ses propos sont parfois difficiles à entendre. Il nous dira notamment lorsque je l'interroge sur son emploi du temps de la veille : "Ce que j'ai fait hier? Oui, j'ai déjeuné sur le coup de midi, je suis allé rechercher mon fils parce qu'il n'a pas encore son permis de conduire, heu puis... j'ai été faire des courses, j'ai été faire des courses et c'est à peu près tout ». La nostalgie d'un temps révolu est présente dans son discours et il valorise l'emploi dans ses propos: "Je savais qui j'étais avant, tout était beaucoup plus simple, quoi, ma place dans la société. Je savais que j'étais ouvrier et que je devais me plier aux exigences du contremaître; eh bien la hiérarchie, maintenant, je n'y arrive plus, je ne sais plus où je suis dedans ", ou encore "Le travail, c'était aussi une place dans la société. J'étais ouvrier et fier de l'être... » La déstructuration des trajectoires familiales est clairement à imputer à cette incapacité à regagner le marché du travail dans un contexte de chômage massif: Louis n’a pas de formation, aucune qualification et aucune compétence sur laquelle s'appuyer pour rebondir. Au moment de l'entretien, Louis a 45 ans. Les derniers propos de l'entretien sont également très éloquents :

- Vous avez des projets pour l'avenir?

- Quels projets?

- Je ne sais pas, je vous demande, non?

- Non, vieillir.

La rencontre avec Stéphane se fait quelques jours après et se déroule de manière assez déconcertante, parce que je m’attends à trouver la même désespérance familiale. Mais Stéphane est joyeux, plein d'entrain et assez décontracté. Il me raconte son décrochage scolaire : « l'école, c'était pas mon truc... ». Il n'a pas envie de se former ou d'apprendre : " de toute façon, je n'en ai plus besoin, je pars demain pour un chouette boulot à l'étranger ». Suivra une longue discussion où Stéphane me racontera qu'il vient d'être engagé dans une station de ski prestigieuse par un club tout aussi prestigieux. Il ne sera pas professeur de ski, il n’a jamais skié de sa vie, mais animateur dans la station. Il me quitte en disant qu’il doit préparer ses bagages. Quelques jours plus tard, par hasard, je croise Stéphane dans un centre commercial... Il ne partira jamais. 


\section{CONCLUSION}

Les recherches scientifiques portant sur le devenir des enfants issus de familles où le chômage s'est installé durablement ont mis en avant, dès les années 1990, l'importance des ressources disponibles dans le ménage : ressources financières, psychologiques et sociales qui peuvent soit se combiner pour permettre un dépassement de la situation de crise née de la perte d'emploi, soit se révélées insuffisantes pour y faire face. Dans ce dernier cas, les conséquences du chômage de longue durée rejaillissent sur la génération suivante : la souffrance parentale envahit la sphère familiale.

Cet article montre combien ce premier niveau d'analyse doit se combiner avec un second, afin de pouvoir saisir la complexité des transmissions générationnelles. La capacité de nouer des relations de confiance interpersonnelles solides et durables avec un tiers peut en effet permettre au jeune de construire son devenir en référence à ce modèle normatif tiers et ainsi lui permettre de sortir du cercle familial enfermant, structuré autour de l’échec professionnel.

Mais cet article permet également de reposer la question centrale, épinglée depuis les travaux de l'équipe de Lazarsfeld dans les années 1930, de la fonction sociale de l'emploi : « dismoi ce que tu fais, je te dirai qui tu es ». En effet, l'expérience du chômage de longue durée s'inscrit aujourd'hui au cœur de mutations culturelles contemporaines. On assiste actuellement à une forme de concurrence entre deux modèles normatifs dominants : le premier repose sur un investissement identitaire unicentré autour de l'emploi, le second davantage sur une forme de polycentration identitaire où l'emploi ne constitue plus qu'un socle possible au cœur d'une quête d'épanouissement personnel et de réalisation de soi. Cette capacité à investir d'autres sphères, d'autres univers de sens que ceux construits autour de l'activité professionnelle permettent alors de relativiser la perte d'emploi et de pouvoir assurer, dans une même logique de polycentration, les mécanismes de transmission. À l'inverse, l'individu qui ne parvient pas à surmonter l'épreuve du chômage demeure englué dans une relation trop fusionnelle à l'emploi. Les logiques de transmission s'en ressentent, le climat familial se détériore et le jeune doit trouver ailleurs les ressources nécessaires à la structuration de son univers de vie. 


\section{Bibliographie}

Benoît-Guilbot, O.,et,D. Gallie (1992). Chômeurs de longue durée, Arles, Actes Sud.

BRoccolochi, S., C. CESAR, et B. LARGUEZE (2004). Qu'est-ce qui désavantage scolairement des enfants de familles défavorisées socialement?, Cresas - inrp, Recherche et Sociétés.

Bruniaux, C., et B. Galtier (2003). «L'étude du devenir des enfants pauvres de familles défavorisées : l'apport des expériences américaine et britannique », Les papiers du Cerc.

CERC (2004), Les Enfants Pauvres en France, Rapport no 4.

CHRISTOFFERSEN, M.N. (1994). «A follow-up study of longterm Effetcs of Unemployment on Children: loss of Self-esteem and Self-destructive Behavior among Adolescents», Childhood, $\mathrm{n}^{0}$ 4, p. 212-220.

Clark, L., J. Grusec, C.R. Jones, G. Plickert, et L. Tepperman (2002). «La pauvreté, le capital social, les compétences parentales et les résultats des enfants au Canada », Human Resources Development Canada, mars 2002.

CORAK, M. (2004). Les enfants pauvres deviennent-ils des adultes pauvres?, Ministère jeunesse éducation recherche/Carré des Sciences.

CuRTIS, L., et S. Phipps (2000). «Impact des ressources économiques sur l'état de santé des enfants et leur succès scolaire », Human Resources Development Canada.

DAVAillon, A., et E. NAUZE-FicheT (2004). « Les trajectoires scolaires des enfants pauvres », Education et formations, $\mathrm{n}^{\circ} 70$.

Delcroix, C. (1995). "Des récits de vie croisés aux histoires de famille», Current Sociology/Sociologie contemporaine, $\mathrm{n}^{\mathrm{0}}$ 43, p. 61-67.

Dronkers, J. (1994). «Fathers' Unemployment and Children's Academic Achievement», Education and Society, $\mathrm{n}^{\mathrm{O}}$ 12, p. 3-13.

DuÉE, M. (2004). «L'impact du chômage des parents sur le devenir scolaire des enfants », Série des documents de travail de la Direction des Études et Synthèses économiques, Insee.

EisenBerg, P., et P.F. LAZARSFeld (1938). «The psychological Effects of Unemployment», Psychological Bulletin, ${ }^{0}$ 35, p. 358-390.

Gallie, D., C. Marsh, et C. Vogler (1994). Social Change and the Experience of Unemployment, Oxford, Oxford Press University.

GIDDENS, A. (1994). Les conséquences de la modernité, Théorie sociale contemporaine, Paris, L'Harmattan.

HEPWORTH, S.J. (1980). «Moderating actors of the psychological impact of unemployment», Journal of Occupational Psychology, $n^{0}$ 53, p. 139-148. 
JAHODA, M. (1979). «The impact of unemployment in the 1930s and the 1970s», Bulletin of the British Psychological Society, $\mathrm{n}^{0}$ 32, p. 309-314.

Jamoulle, P. (2002). La débrouille des familles, Bruxelles, De Boeck.

LAHIRE, B. (1995). Tableaux de famille, Heurts et malheurs scolaires en milieu populaire, Paris, Seuil.

LAHIRE, B. (1996). «La variation des contextes en sciences sociales, Remarques épistémologiques », Annales HSS, n² 2, p. 381-407.

LAZARsfeld, P., M. JAhOdA, et H. Zeisel (1981). Les chômeurs de Marienthal, Paris, Les éditions de Minuit.

LEWIS, O. (1986). Les enfants de Sanchez : autobiographie d'une famille mexicaine, Paris, Gallimard.

LOPEZ, A. (2004). «Autour de l'insertion professionnelle : anciennes et nouvelles lignées de pauvreté », Publication du CERC.

Lopez, A., et G. Thomas (2004). "Précarités vécues dans l'enfance et insertion professionnelle », Publication du Cerc.

McKee-Ryan, F.M., Z. Song, C.R. WAnberg, et A.J. KinicKi (2005). «Psychological and Physical Well-being during Unemployment: a meta-analytic Study», Journal of Applied Psychology, $\mathrm{n}^{\mathrm{o}}$ 90, p. 53-76.

Paugam, S. (1993). « Famille et vie conjugale », Document du CERC, nº 109.

Reinhardt Pedersen C., et M. MAdSEN (2002). «Parents' labour market participation as a predictor of children's health and wellbeing: a comparative study in five Nordic countries», Journal of Epidemiology, Community Health, nº 56, p. 861-867.

Ricoeur, P. (1990). Soi même comme un autre, Paris, Seuil.

RoQues, M. (1995). Sortir du chômage, Liège, Mardaga.

TASHAKKORI, A., et C. TEDDIE (1998). Mixed methodology, Combining Qualitative and Quantitative Approaches, London, Sage, Social Research Methods Series, vol. 46. 